\title{
Essential Echocardiography: Transesophageal Echocardiography for Non-Cardiac Anesthesiologists
}

\author{
Timothy M. Maus, Sonia Nhieu, Seth T. Herway (Editors). Springer; \$99.00 USD (eBook) \\ or \$129.00 USD (softcover); pages: 301. Includes 127 online echocardiography videos. \\ ISBN: 978-3-319-34122-4
}

\author{
Elizabeth Clarke Miller, MD
}

Received: 16 December 2016/Accepted: 16 January 2017/Published online: 30 January 2017

(C) Canadian Anesthesiologists' Society 2017

Since the introduction of cardiac ultrasonography in 1953, anesthesiologists have been leaders in advancing applications for it in perioperative, intraoperative, and critical care settings. Drs Maus et al., in their new book, Essential Cardiography: Transesophageal Echocardiography for Non-Cardiac Anesthesiologists, provide a comprehensive, up-to-date overview of this important area of growth in our specialty. The authors highlight the need for perioperative physicians to acquire basic skills in echocardiography to monitor patients' cardiac function, assess responsiveness to pharmacological or volume resuscitation interventions, and diagnose the causes of hemodynamic instability. This text provides an outstanding introduction for physicians in this area.

A particularly unique aspect of Maus et al.'s book is that it fills a niche wherein anesthesiologists and trainees can be educated in the basic aspects of transesophageal echocardiography (TEE) for non-diagnostic and monitoring purposes, as well as for indications outside the cardiac operating room. Thus, it is directed to a majority of anesthesia practitioners, which is not the case with many other echocardiography texts that have targeted advanced National Board of Echocardiography-trained physicians who use TEE diagnostically and to direct medical or surgical cardiac interventions.

Although this text is geared toward the more basic applications of TEE, it is appropriately comprehensive and provides substantive descriptions. Maus et al. skillfully created a fine balance in providing an in-depth, detailed

E. C. Miller, MD ( $\square)$

The Ottawa Hospital, University of Ottawa, Ottawa, ON, Canada

e-mail: emill015@uottawa.ca reference manual that is still readable and makes challenging concepts easy to grasp. For example, the descriptions of how best to maneuver the ultrasound probe (Chapter 1) or the anatomic associations between various cardiac structures (Chapter 2) - e.g., the "wrapping around" nature of the right heart - are straightforward and keep the reader engaged. Chapter 3, on Basic Ultrasound Physics, Doppler Ultrasound, and Hemodynamic Assessment, describes the challenging aspects and the equations of the physics of ultrasound in easily understandable terms. The information is presented clearly, and corresponding illustrations are used to relate the material realistically to everyday applications. The topics of aliasing and the Nyquist limit, which novices may find difficult to grasp, are expertly presented using a description and image of a "Wagon Wheel".

Other TEE topics covered in the manual include certification, indications, contraindications, probe movements, essential views, assessment of left and right ventricular function, valve imaging, diastology, thoracic aortic imaging, rescue echocardiography for assessing hemodynamic instability, common misdiagnoses, and imaging for adult congenital heart disease. Also included are supporting tables that outline and summarize, with representative images, the components of both basic and comprehensive TEE examinations. It would be helpful to bookmark these tables for quick reference when practicing or reviewing echocardiography images in the ultrasound reading room.

Other chapters describe surface ultrasound for transthoracic echocardiography (TTE) as well as for vascular access. A limitation of these final chapters is that they are quite basic when compared with the chapters on TEE. Although they provide a simple 
overview, they are insufficiently detailed to be considered as stand-alone reference material for someone attempting to learn point-of-care ultrasonography, TTE, or detailed hemodynamic assessments using surface ultrasound.

My favourite aspect of this book is its beautiful images. Each TEE view is presented with a clear, well-labeled photograph of an ultrasound image. As well, there are references to video echocardiography loops (found on the Springer website) that correspond to each image. Video loops are an essential component of any ultrasound reference, and they add a great deal to the utility of this book. That said, the details of how to view these loops could have been better described in the text (they required some extra searching for more details). One can access the videos by locating the book using the ISBN number on the Springer website (http://link.springer.com/book/10.1007/ 978-3-319-34124-8), a link that presents a breakdown of each chapter in the book. After clicking on a chapter of interest, a dropdown menu of "supplementary material" is presented. Various videos can be easily downloaded from this dropdown menu. These downloads provide an important addition to the printed images.
Overall, Essential Echocardiography fills several purposes. As it is written by cardiac and non-cardiac anesthesiologists, it provides a balanced perspective. Hence, it serves as a practical initial reference source for the anesthesiologist wishing to obtain basic TEE skills or to prepare for TEE courses or a basic PTE examination. The material in Essential Echocardiography is a guide for practicing TEE in the TEE simulator or in the ultrasound reading room. It would be an exceptional resource for anesthesia residents who are learning TEE as a part of their cardiac anesthesia rotations and for non-cardiac anesthesia echocardiography fellows who are training in basic TEE. Essential Echocardiography is an enjoyable cover-to-cover read and should be recommended to any non-cardiac anesthesiologist or anesthesia trainee.

Conflicts of interest None declared.

Editorial responsibility This submission was handled by Dr. Hilary P. Grocott, Editor-in-Chief, Canadian Journal of Anesthesia. 